\title{
Prediction of bulk tank somatic cell count violations based on monthly individual cow somatic cell count data
}

\author{
V. Fauteux, ${ }^{* 1}$ E. Bouchard, ${ }^{*}$ D. Haine, ${ }^{*}$ D. T. Scholl, $†$ and J. P. Roy* \\ *Faculté de médecine vétérinaire, Université de Montréal, 3200 rue Sicotte, CP 5000, Saint-Hyacinthe, Québec, Canada, J2S 2 M2 \\ †South Dakota Agricultural Experiment Station, South Dakota State University, Brookings 57007-1898
}

\begin{abstract}
The regulatory limit in Canada for bulk tank somatic cell count (BTSCC) was recently lowered from 500,000 to 400,000 cells $/ \mathrm{mL}$. Herd indices based on changes in cow somatic cell count over 2 consecutive months (e.g., proportion of healthy or chronically infected cows, cows cured, and new intramammary infection rate) could be used as predictors for BTSCC violations. The objective of this study was to develop a predictive model for exceeding the limit of 400,000 cells/mL in the next month using these herd indices. Dairy Herd Improvement (DHI) data were used from 924 dairy herds in Québec, Canada. Test-day BTSCC was estimated by dividing the sum of all cows' DHI test-day somatic cell count times DHI test-day milk production by the total volume of milk produced by the herd on that test-day. In total, 986 of $8,681(11.4 \%)$ estimated BTSCC exceeded 400,000 cells $/ \mathrm{mL}$. The final predictive model included 6 variables: mean herd somatic cell score at the current test-month, proportion of cows $>500,000$ cells/mL at the current test-month, proportion of healthy cows during lactation at the current testmonth, proportion of chronically infected cows at the current test-month, average days in milk at the current test-month, and annual mean daily milk production. The optimized sensitivity and specificity of the model were 76 and $74 \%$, respectively. The positive predictive value and negative predictive value were 25 and $95 \%$, respectively. This low positive predictive value and high negative predictive value demonstrated that the model was less accurate at predicting herds that would violate the estimated BTSCC threshold but very accurate at identifying herds that would not. In addition, the area under the curve for the receiver operating characteristic curve was 0.82 , suggesting that the model had excellent discrimination between test-months that did and did
\end{abstract}

Received January 20, 2014

Accepted January 6, 2015.

${ }^{1}$ Corresponding author: veronique.fauteux@umontreal.ca not exceed 400,000 cells/mL. An internal validation was completed using a bootstrapped resampling-based estimation method and confirmed that the final model provided a validated estimate of predictive accuracy. This model could be used to monitor and advise clients on impending risks of exceeding the BTSCC limit.

Key words: bulk tank, somatic cell count, prediction model, violation, dairy herd

\section{INTRODUCTION}

Milk quality influences the overall success of a dairy farm. In Canada, the Canadian Dairy Commission is responsible for developing quality standards for milk production (Canadian Dairy Commission, 2013). Failure to comply with these standards results in financial penalties and ultimately cessation of milk shipments to the processing plant (Les Producteurs de Lait du Québec, 2013). The regulatory limit for bulk tank SCC (BTSCC) on all Canadian dairy farms was recently lowered, as part of an initiative with the Canadian Quality Milk program, from 500,000 to 400,000 cells/ $\mathrm{mL}$ (Les Producteurs de Lait du Québec, 2014).

Several strategies are available to dairy producers and advisors to help manage and monitor BTSCC. Hand et al. (2012) suggested that participation in DHI programs, wherein individual cow milk samples are frequently tested for SCC, could be used to monitor and improve BTSCC. Monthly SCC data are routinely interpreted by bovine practitioners to evaluate the udder health status of their client herds. To facilitate this practice, new dairy herd health management software has recently been developed and is being used in Québec, Canada [DSAHR (http://www.dsahr.ca/Liens/ PageLiens.aspx) and CCStat program software]. Initially, however, region-specific benchmark data needed to be generated for several of the monitored indices to enhance the performance of the software for udder health assessment in Québec dairy herds. Results from this recent benchmark study confirmed that herds with high mean annual SCC had fewer cows with healthy udders and more cows with chronically infected ud- 
ders during lactation compared with low SCC herds (Fauteux et al., 2014).Thus, one could speculate that these herd-level udder health indices could be used as predictors for possible BTSCC violations under the new cutoff of 400,000 cells/mL.

Lukas et al. (2008) demonstrated that monthly mean and standard deviation (SD) of monthly BTSCC data can be used to estimate the probability of exceeding 5 different SCC levels, ranging from 200,000 to 600,000 cells $/ \mathrm{mL}$, in the subsequent month. Specifically, it was documented that the probability of a specific BTSCC violation was greater as monthly mean and SD BTSCC increased, and that high BTSCC herds had less predictable performance because their monthly BTSCC were usually more variable (Lukas at al., 2008). This research team also developed logistic regression models that demonstrated the odds of exceeding a BTSCC standard were significantly greater in the summer months and for smaller herds. Furthermore, Lievaart et al. (2010) reported that data on herd characteristics, season, management, and monthly SCC could be used to correctly predict more than $70 \%$ of the subsequent month herd SCC within a narrow range of 20,000 to 30,000 cells $/ \mathrm{mL}$. Hence, predictive models can be useful tools to assist with udder health management assessments and decisions. The objective of this study was to develop a predictive model of exceeding a BTSCC limit of 400,000 cells/mL for dairy farms in Québec, Canada.

\section{MATERIALS AND METHODS}

\section{Herds and Data}

Individual cow DHI data were used of 924 Québec, Canada, dairy herds from October 1, 2007, to December 31, 2008. To be eligible for inclusion in the study, herds had to be monitored at least on a monthly basis by their herd veterinarian using the DSAHR software, had to be enrolled in the DHI milk recording program, and had to have completed at least 6 DHI tests during the 2008 calendar year. Moreover, all participating herds had to have a minimum average herd size of 30 cows in lactation. Herd udder health indices were calculated using data from 2 consecutive DHI tests, and the interval between these 2 tests had to be $\leq 50 \mathrm{~d}$. Data from 4 successive test-months were used to construct the predictive model: 3 predictive test-months and 1 predicted test-month. Therefore, herds had to present with at least 4 consecutive DHI test-months, each separated by $\leq 50 \mathrm{~d}$, to be included in the database. Herd selection is summarized in Figure 1. The final database included primarily Holstein cows, along with some Jersey, Ayrshire, and Brown Swiss cows.

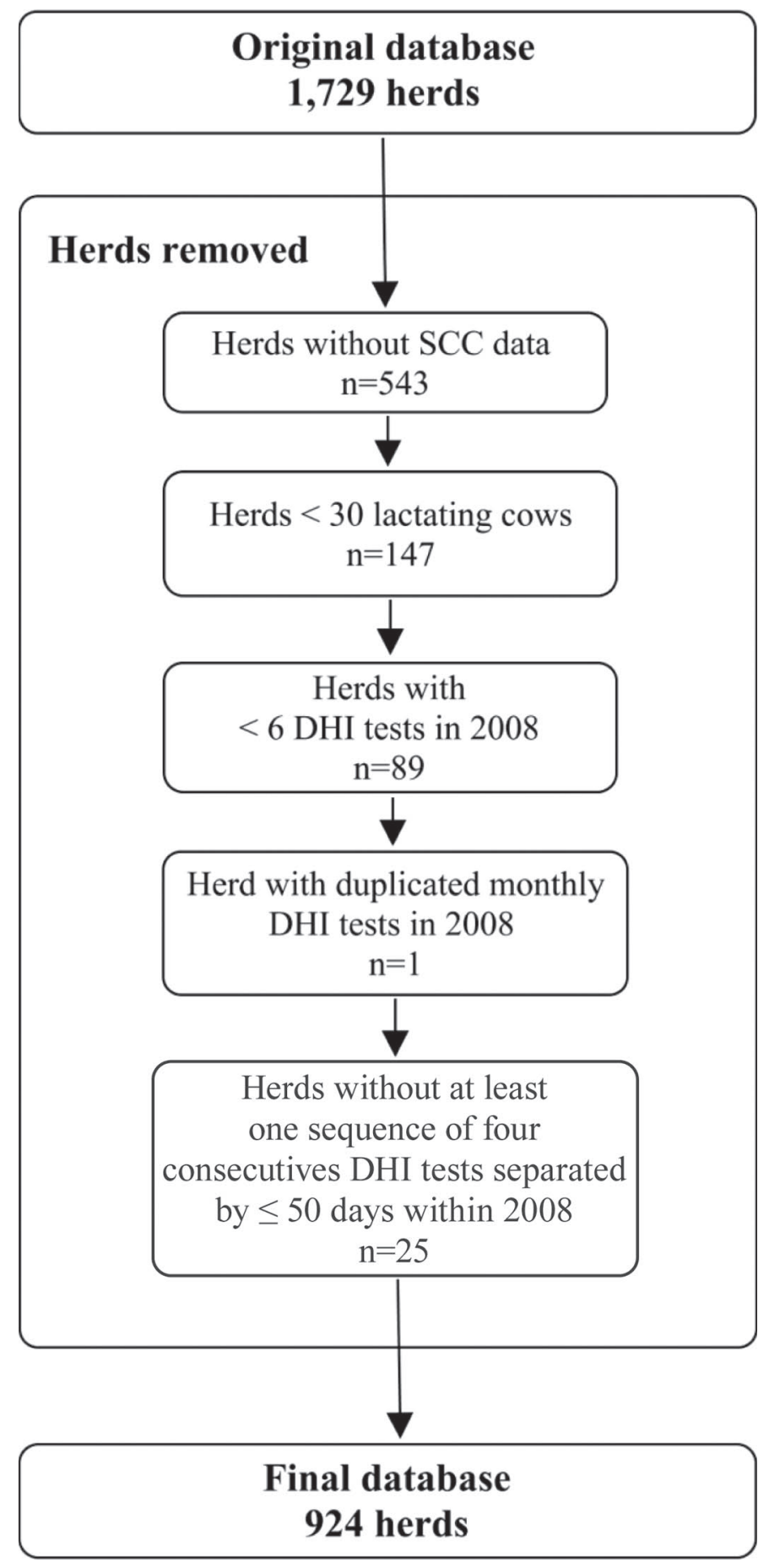

Figure 1. Summary of the herd selection process performed before the construction of a model to predict 400,000 cells/mL-estimated bulk tank SCC violations using individual cow DHI data from 924 dairy herds in Quebec, Canada, between October 1, 2007, and December 31, 2008. 


\section{Model Construction}

Model Basis. A logistic regression model was constructed to predict the odds of an estimated BTSCC (EBTSCC) violation in the next test-month (testmonth+1), based on a cut-off value of 400,000 cells/ $\mathrm{mL}$. The EBTSCC was calculated as follows: $\Sigma$ (individual cow DHI test-day SCC $\times$ DHI test-day milk production)/total volume of milk produced by the herd on that given test-day (Lievaart at al., 2010). Each EBTSCC was subsequently dichotomized as either $\leq 400,000$ or $>400,000$ cells $/ \mathrm{mL}$. Herd was included in the model as a random effect. A list of predictor variables considered for inclusion as fixed effects in the model is presented in Table 1. It is important to note that SCC is an indicator of intramammary inflammation, not infection. In this study, it was used as a proxy of intramammary infection using a cut-off level of 200,000 cells/mL (Dohoo and Leslie 1991). A specific index representing the previous 3-mo EBTSCC trend of each herd was constructed using a linear regression model (Table 1). The slope of the resulting linear regression model was used to estimate whether the EBTSCC was increasing, decreasing, or remaining stable in the previous 3 mo.

Predictive Model Fitting. First, correlation among predictor variables was determined to identify clusters of correlated variables and to reduce the likelihood of overfitting the model. A correlogram was generated and predictor variables with Spearman rho correlation coefficients $>0.8$ were considered highly correlated. Principal component analysis (PCA) was also conducted to explore the data for clusters of correlated variables. Principal component analysis uses orthogonal transformations of the data to identify sets of linearly uncorrelated variables (Joliffe and Morgan, 1992). Principal component analysis was used to discard redundant variables, as described by Joliffe (1972), McCabe (1984), and Krzanowski (1987). Thus, PCA allows for clusters of variation within the data set to be identified.

Lasso (least absolute shrinkage and selection operator) regression (Tibshirani, 1996) was subsequently applied on the 3 clusters identified by PCA (Avalos, 2009). Lasso regression is used to identify irrelevant predictor variables in a linear regression model by applying a $\mathrm{L}_{1}$-type penalty to the regression coefficients. As a result of this process, a smaller subset of predictor variables with the strongest effects can be determined (Tibshirani, 1996).

The remaining predictor variables were then offered to a logistic regression model for EBTSCC violation with repeated measures. A manual backward elimination procedure was used to build the final model. The full and nested models were compared using the likelihood ratio test. Statistical significance was declared at $P<0.05$.

Predictive Model Evaluation. Nonlinear terms and interaction terms with mean yearly production were introduced into the model to evaluate the model assumptions of linearity between the outcome and continuous predictor variables and additivity of effects. Residuals for the different predictor profiles were also visually examined. The slope of the linear predictor in the logistic regression model was used to assess the calibration of the model predictions (Miller et al., 1991; Harrell et al., 1996). This represents the agreement between the predicted and the observed risk of exceeding 400,000 cells/mL (Harrell et al., 1996). Calibration was also visually assessed using a Lowess smoother function of predicted and observed probabilities. A $45^{\circ}$ line with an intercept at 0 and a slope of 1 represents a perfect match. The slope of the regression line, fitted to the logits of the predicted probabilities and the observed proportions, is a measure of the direction and spread of the predicted probabilities (Cox, 1958). Conversely, the intercept is a measure of the overall bias of the model (Cox, 1958). Model discrimination between violation outcomes was assessed by area under curve (AUC) for the receiver operating characteristic (ROC) curve (Hosmer and Lemeshow, 2000). The discriminatory ability of the predictive model was estimated using the $c$ index (Harrell et al., 1996), which is equivalent to the AUC for the ROC curve for binary outcomes (Harrell et al., 1996; Hosmer et al., 2013). The AUC measures a predictive model's ability to discriminate between those subjects (herds in this case) that experience the outcome of interest (EBTSCC exceeding 400,000 cells/ $\mathrm{mL}$ ) versus those that do not. The AUC ranged from 0.5 to 1 (Hosmer et al., 2013).

Finally, internal validation was performed by calculating the expected optimism of the AUC for the ROC curve with a bootstrap resampling procedure (Harrell, 2001; Steyerberg et al., 2001). Optimism was computed by drawing 200 same-size random bootstrap samples with replacement from the data set and then applying the model to each of the bootstrap samples (Efron and Tibshirani, 1993). Optimism is the difference between the AUC for the ROC curve of models developed in each of the bootstrap samples and the AUC in the original data set (Harrell et al., 1996). The validated estimate of predictive accuracy was determined by subtracting the optimism from the original AUC. All analyses were completed with R version 3.1.0 [R Core Team, 2012; packages: FactoMineR (Husson et al., 2014), glmnet (Friedman et al., 2010), and lme4 (Bates et al., 2014; D. Bates, unpublished data)]. 
Table 1. Predictor variables considered for inclusion in the logistic regression model to predict the odds of a 400,000 cells/mL estimated bulk tank SCC (EBTSCC) violation in the next test-month for 924 dairy herds in Quebec, Canada, in 2008

\begin{tabular}{|c|c|}
\hline Predictor variable & Definition \\
\hline $\begin{array}{l}\text { Mean annual number of lactating cows in herd } \\
\text { Mean annual herd parity } \\
\text { Mean herd annual daily milk production } \\
\text { Mean daily milk production at the } 2 \text { previous test-months }{ }^{1} \\
\text { Mean DIM at the } 2 \text { previous test-months } \\
\text { Mean individual SCS }{ }^{2} \text { at the } 2 \text { previous test-months } \\
\text { Proportion of healthy cows during lactation at the } 2 \text { previous test-months } \\
\text { Proportion of chronically infected cows during lactation at the } 2 \text { previous test-months } \\
\text { Proportion of cured cows during lactation at the } 2 \text { previous test-months } \\
\text { Proportion of newly infected cows during lactation at the } 2 \text { previous test-months } \\
\text { Proportion of cows } \geq 1,000,000 \text { cells } / \mathrm{mL} \text { at the } 2 \text { previous test-months } \\
\text { Proportion of cows } \geq 500,000 \text { cells } / \mathrm{mL} \text { at the } 2 \text { previous test-months } \\
\text { Proportion of cows } \geq 200,000 \text { cells } / \mathrm{mL} \text { at the } 2 \text { previous test-months } \\
\text { EBTSCC trend } \\
\text { Season of the test-month }\end{array}$ & 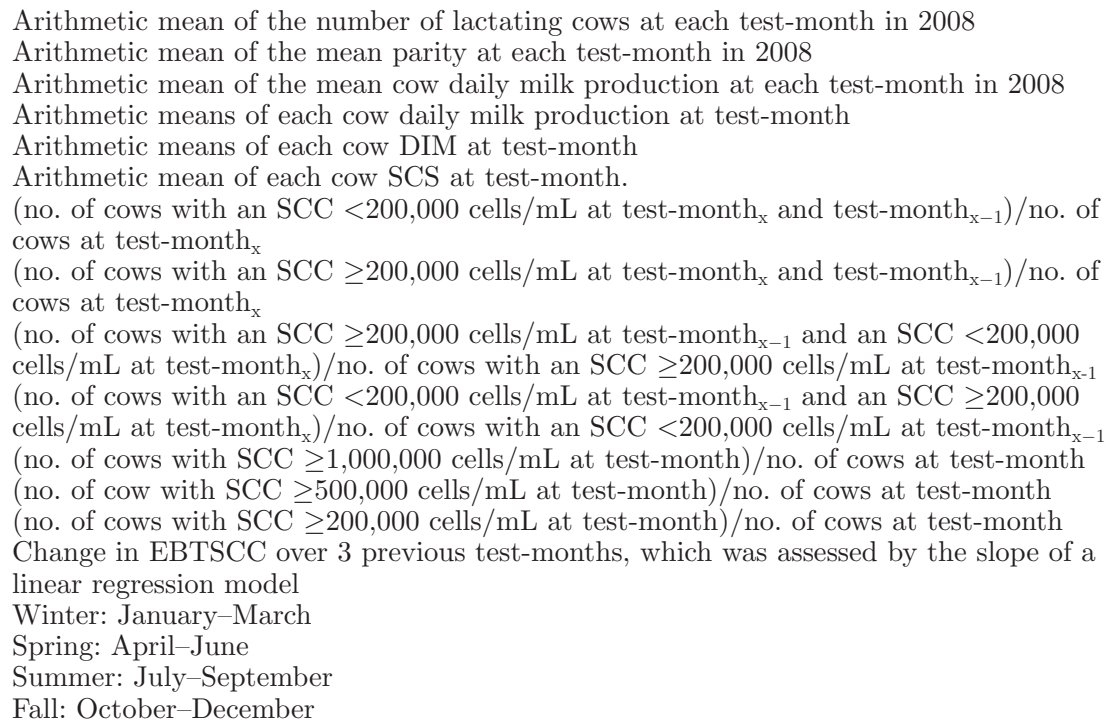 \\
\hline
\end{tabular}

产 ${ }^{1}$ Two previous test-months $=$ test-month -1 and test-month -2 before the predicted test-month, which corresponded to test-month +1 .

$\stackrel{2}{2}$ SCS was calculated by transforming individual SCC using the following formula: $\log _{2}(\mathrm{SCC} / 100)+3$, where SCC was measured by 1,000 cells/mL (Ali and Shook, 1980$)$. 


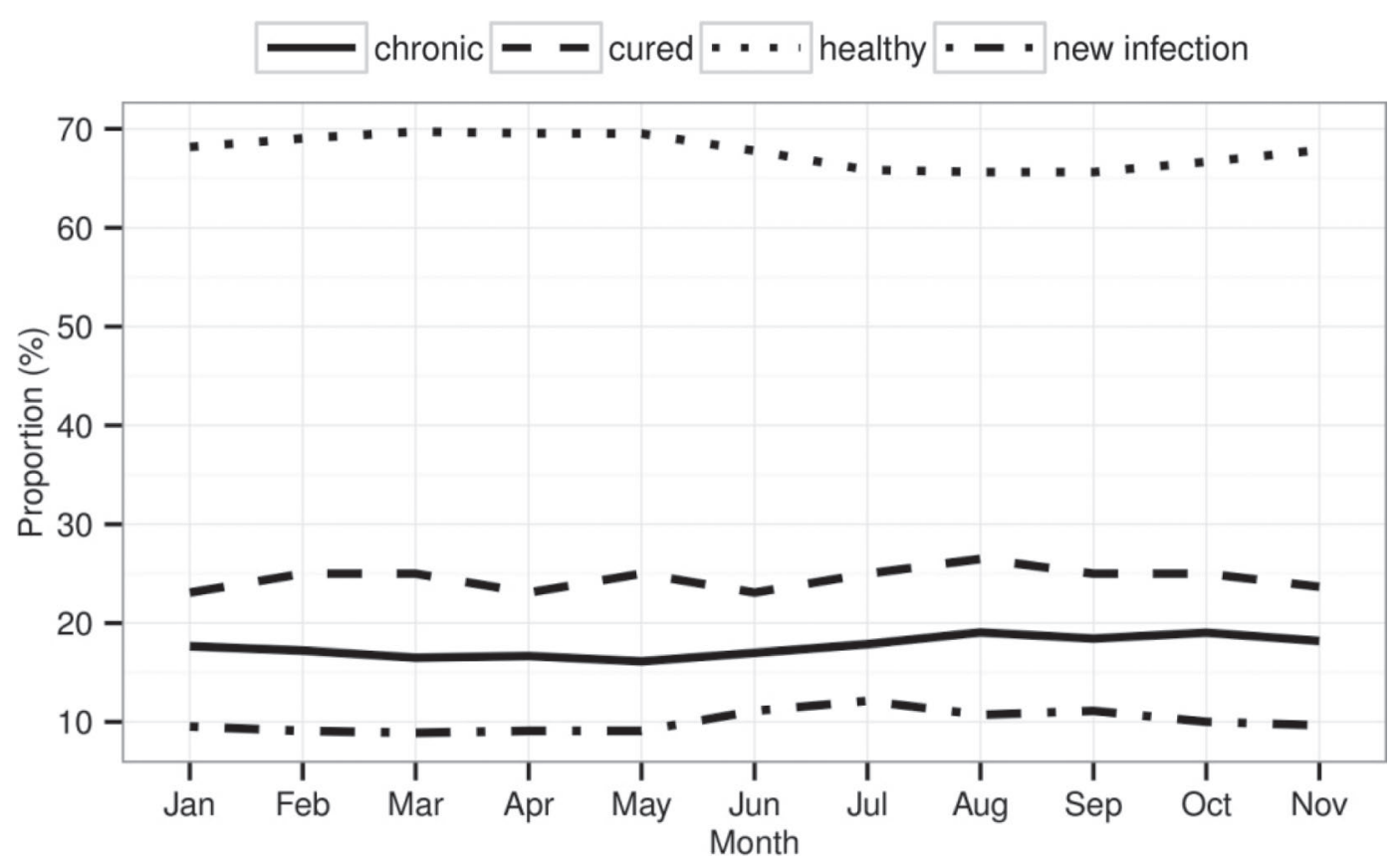

Figure 2. Monthly mean proportion of healthy, cured, newly infected, and chronically infected cows based on 2 consecutive DHI test-months for 924 dairy herds in Quebec, Canada, during 2008. A threshold of 200,000 cells/mL was used as the definition of intramammary infection.

\section{RESULTS}

\section{Descriptive Statistics}

Descriptive statistics for annual EBTSCC, herd SCC at test-month, SCS at test-month, daily production by cow, and mean number of cows at each test-month are presented in Table 2. Overall, there were 986 observations $(11.4 \%)$ with EBTSCC $>400,000$ cells $/ \mathrm{mL}$ and 7,695 observations (88.6\%) with EBTSCC $\leq 400,000$ cells/mL. Mean EBTSCC was 251,000 cells/mL.

Figure 2 and Figure 3 show that the proportion of healthy, cured, newly infected, and chronically infected cows during 2008 and the monthly proportion of SCC measures exceeding 1,000,000 cells/mL, 500,000 cells/ $\mathrm{mL}$ and 200,000 cells/mL for the same year varied by less than $10 \%$ during the year.

\section{The Logistic Regression Model}

Twenty-five predictor variables were evaluated with correlation analyses (Pearson correlation coefficient, PCA and Lasso regression); subsequently, 18 variables were excluded because of collinearity. The Pearson correlation coefficients between each pair of the 25 variables are presented in Supplemental Table S1 (http://dx/doi. org $/ 10.3168 / j d s .2014-7965)$. Season of test-month was excluded as a result of the correlation analyses. The full model included the following variables: mean individual SCS at test-month -1 , proportion of cows $>500,000$ cells $/ \mathrm{mL}$ at test-month -1 , mean herd annual daily milk production per cow, average DIM at test-month -1 , proportion of healthy cows at test-month -1 , proportion of chronic cows at test-month -1 , and EBTSCC trend. The EBTSCC trend was removed from the

Table 2. Descriptive statistics on 924 dairy herds in Quebec, Canada: Individual cow DHI data from October 1, 2007, to December 31, 2008, were obtained for these herds and used to construct a 400,000-cells $/ \mathrm{mL}$ estimated bulk tank SCC (EBTSCC) violation predictive model

\begin{tabular}{lccccccc}
\hline & \multicolumn{2}{c}{$\begin{array}{c}\text { No. of } \\
\text { test-month } \\
\text { Item }\end{array}$} & & & \multicolumn{3}{c}{ Percentile } \\
\cline { 5 - 8 } & data & Mean & SD & & 25 th & 50 th & 75 th \\
\hline EBTSCC $(\times 1,000$ cells $/ \mathrm{mL})$ & 8,681 & 251 & 125 & 161 & 227 & 317 \\
Mean herd SCC at test-month & 9,605 & 269 & 141 & 172 & 243 & 336 \\
Mean herd SCS at test-month & 9,605 & 2.8 & 0.60 & 2.4 & 2.8 & 3.2 \\
Mean daily production by cow $(\mathrm{kg})$ & 9,605 & 29.3 & 3.91 & 26.8 & 29.4 & 31.9 \\
Mean no. of lactating cows at test-month & 8,681 & 55.3 & 29.26 & 39.0 & 49.0 & 62.0 \\
\hline
\end{tabular}




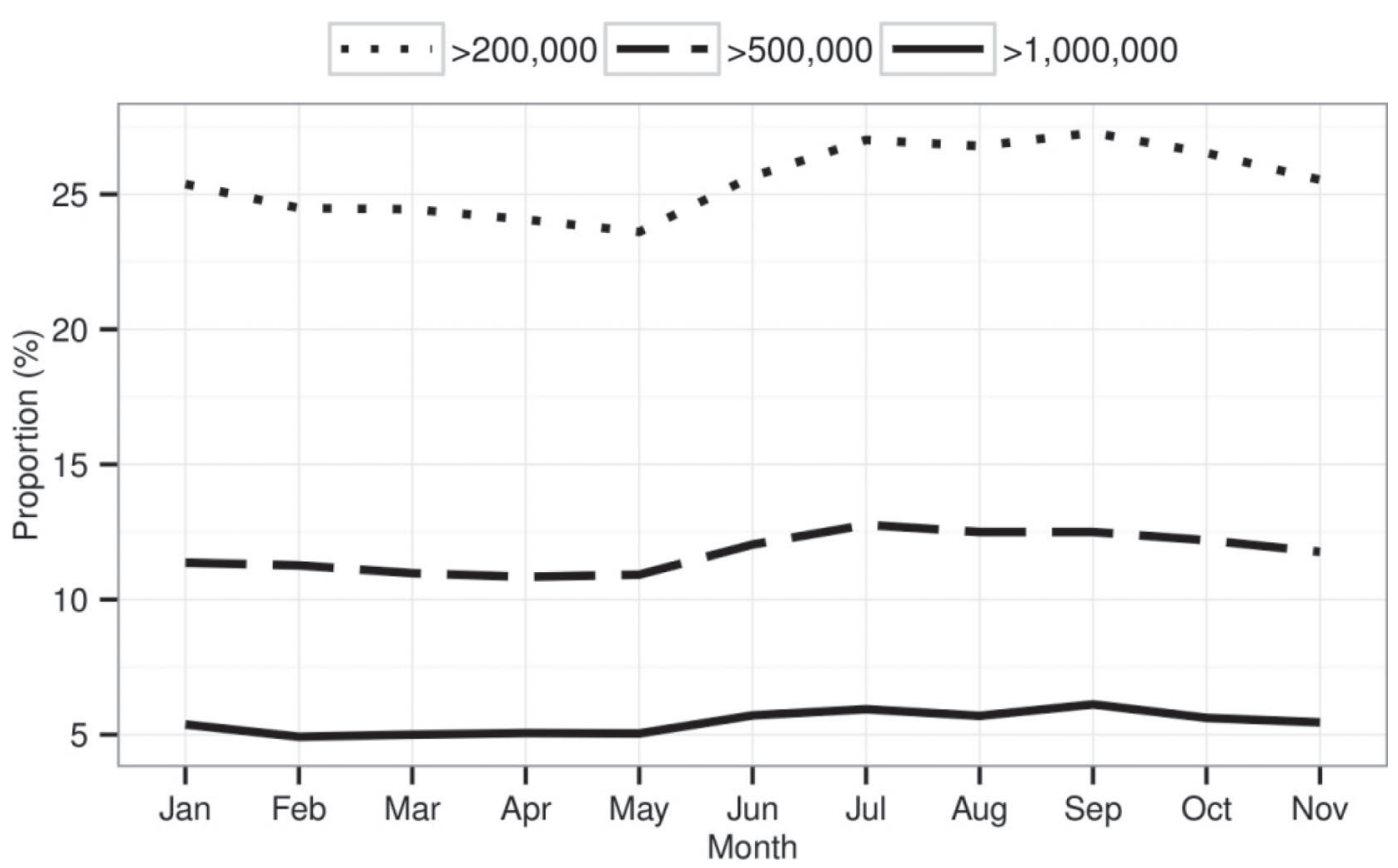

Figure 3. Mean monthly proportion of individual cow SCC exceeding 1,000,000, 500,000, and 200,000 cells/mL for 924 dairy herds in Quebec, Canada, during 2008.

model because it was not significantly associated with the probability of EBTSCC exceeding 400,000 cells/ $\mathrm{mL}$. The other 6 predictor variables were retained in the model. No statistically significant interaction terms were identified. Quadratic terms for the proportion of cows $\mathrm{SCC} \geq 500,000$ cells $/ \mathrm{mL}$ at test-month -1 , the proportion of healthy cows at test-month -1 , and mean individual cow daily production were introduced to the model to meet the assumption of linearity for continuous variables. Final logistic regression model is presented in Table 3 .

\section{Accuracy for Predicting BTSCC Violation}

The accuracy of the model to predict EBTSCC violations using a 400,000 cells/mL threshold was assessed by model calibration and discrimination. Results of the model calibration are presented in Figure 4. The calibration slope for the predictions was estimated at 1.02, and the intercept was relatively close to zero $(0.2$, on a scale from 0 to 1 ). The discriminatory ability of the predictive model is presented in Figure 5. The AUC for the predictive model was 0.82 (95\% CI: $0.81-0.83)$. The optimum cut-point of the ROC curve for distinguishing between predicted violations versus nonviolations for EBTSCC was 0.12. The sensitivity (Se) and the specificity (Sp) optimized at this cut-point were 76 and $74 \%$, respectively. The positive predictive value (PPV) and negative predictive value (NPV) were 27 and $96 \%$, respectively.

Internal validation was conducted using a bootstrap resampling procedure, and the expected optimism of

Table 3. Final logistic regression model predicting a 400,000-cells/mL estimated bulk tank SCC violation based on cow DHI data from October 1, 2007, to December 31, 2008, for 924 dairy herds in Quebec, Canada

\begin{tabular}{|c|c|c|c|c|c|c|}
\hline Item & Estimate & $\mathrm{SE}$ & z-value ${ }^{1}$ & $P$-value & $\begin{array}{l}\text { Odds } \\
\text { ratio }\end{array}$ & $95 \%$ CI \\
\hline (Intercept) & -4.7659 & 0.85798 & -5.55 & $<0.001$ & 0.009 & $0.002-0.046$ \\
\hline Proportion of SCC $\geq 500,000$ cells $/ \mathrm{mL}$ at test-month -1 (squared) & -0.0017 & 0.00061 & -2.75 & 0.006 & 0.998 & $0.997-1.000$ \\
\hline Mean cow daily production (squared) & -0.0011 & 0.00027 & -3.89 & $<0.001$ & 0.999 & $0.998-0.999$ \\
\hline Average DIM at test-month -1 & 0.0037 & 0.00173 & 2.15 & 0.031 & 1.004 & $1.000-1.007$ \\
\hline
\end{tabular}

\footnotetext{
${ }^{1}$ The $\mathrm{z}$-value is the regression coefficient divided by its standard error.
} 


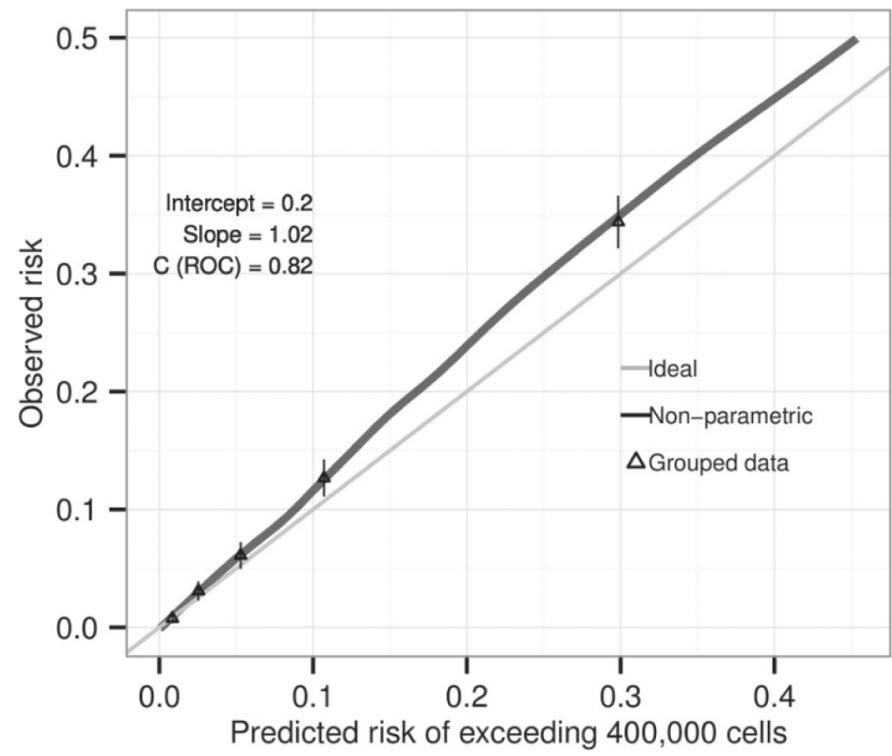

Figure 4. Validation plot for a predictive model of a 400,000 cells mL-estimated bulk tank SCC violation using individual cow DHI data from 924 dairy herds in Quebec, Canada, between October 1, 2007, and December 31, 2008. "Intercept" refers to calibration-in-thelarge, and "slope" refers to the calibration slope for the predictions. "C (ROC)" refers to the C statistic (receiver operator characteristic area under the curve, ROC AUC). The ideal $45^{\circ}$ line has an intercept of 0 and a slope of 1 . Triangles indicate observed risk for quintiles of predictions with $95 \%$ confidence intervals presented as vertical bars.

the final model was less than 0.01 . The same methodology was applied to the full model before it was reduced using a backward selection procedure. The full model had similar optimism of less than 0.01 and AUC for the ROC curve of 0.82 .

\section{DISCUSSION}

\section{Descriptive Statistics}

Mean EBTSCC in the current study was 250,800 cells/mL. Conversely, mean annual BTSCC during 2008 for the 6,601 Québec dairy herds was 257,438 cells $/ \mathrm{mL}$ (Les Producteurs de Lait du Québec, 2009). This difference could be due to several factors, including the fact that EBTSCC were calculated for only a subset of the 6,601 dairy herds in Québec, and that all study herds were enrolled in DHI milk recording. Hand et al. (2012) demonstrated the benefit of being enrolled in a DHI program and having access to cow-level SCC to manage BTSCC. In Québec, during the 2008 calendar year, $5,321(80.6 \%)$ of the 6,601 dairy herds were enrolled in a DHI milk recording program (Les Producteurs de Lait du Québec, 2009). Therefore, the EBTSCC of the study herds may truly be lower than the overall mean BTSCC for Québec dairy herds. On the other hand,

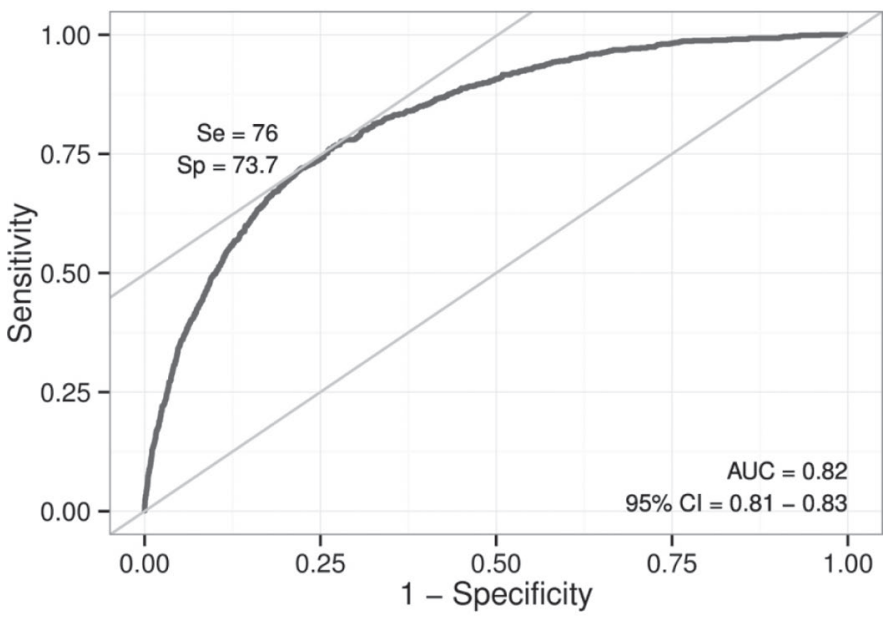

Figure 5. Sensitivity (Se), specificity (Sp), area under the curve (AUC), and 95\% CI for a receiver operating characteristic curve of a predictive model of estimated bulk tank SCC violations using individual cow DHI data from 924 dairy herds in Quebec, Canada, between October 1, 2007, and December 31, 2008.

specific management practices, such as withholding milk from high SCC cows from the bulk tank for calf feeding, could have resulted in BTSCC actually being lower than the corresponding EBTSCC (Lievaart et al., 2007, 2010). Finally, EBTSCC were calculated using only one measurement per month, whereas real monthly BTSCC are calculated using 4 measurements from each month. Increasing the number of measurements would likely have allowed for a more precise estimation of the parameter.

\section{The Logistic Regression Model}

The prediction model for EBTSCC violations has documented that attentive monitoring of general herd udder health status using individual monthly SCC can provide insight into the odds of exceeding the new BTSCC regulatory limit. Moreover, the model demonstrated that mean SCS on DHI test-day was the most important determinant of the probability of exceeding 400,000 cells $/ \mathrm{mL}$ on the next test. These findings suggest that the greatest opportunity for preventing a potential BTSCC threshold violation in subsequent months is monitoring SCS and then intervening when it increases.

Estimated BTSCC was associated with the proportion of cows exceeding 500,000 cells $/ \mathrm{mL}$ and the proportion of chronically infected cows at the current test. Hence, these 2 parameters should also be considered when monitoring BTSCC on farms. Madouasse at al. (2010) established that BTSCC was influenced by the proportion of chronically infected lactating and dry 
cows, new infections over the dry period, and heifers with an SCC $\geq 200,000$ cells $/ \mathrm{mL}$ during the first month of lactation. In the present study, indices relating to the infection dynamics during the dry period and heifer infections were not included in the model because the authors felt that this information was captured by the proportion of cows with SCC $\geq 200,000$ cells/mL at the test-month, and possibly by the proportion of cows with greater than 500,000 or $1,000,000$ cells $/ \mathrm{mL}$. For example, cows and heifers having an SCC $\geq 200,000$ cells $/ \mathrm{mL}$ at first test-month after calving were included in the mean proportion of cows with $\mathrm{SCC} \geq 200,000$ cells $/ \mathrm{mL}$ of the same specific test-month. Hence, it is likely that the indices described by Madouasse et al. (2010) as having an important effect on the BTSCC were similar to those identified in the current study. Indeed, the proportion of chronically infected cows was significantly associated with BTSCC in both studies, and the proportion of cows with SCC >500,000 cells/ $\mathrm{mL}$ at the previous test month provides meaningful information about new infections over the dry period and heifer infections.

\section{Accuracy for Predicting BTSCC Violation}

The estimation of the calibration slope for the prediction demonstrated that the predictive model did not over- or underestimate the odds of EBTSCC exceeding 400,000 cells $/ \mathrm{mL}$. A model that is able to perfectly predict the outcome would be represented by a $45^{\circ}$ line, with an intercept at 0 and a slope of 1 . In the current study, the model intercept was relatively close to zero, suggesting that the model was relatively unbiased at predicting EBTSCC violations (Miller et al., 1993).

The discriminatory ability of the model, represented by the AUC for the predictive model, indicates that the model had excellent discrimination between true EBTSCC violation signals and false EBTSCC violation signals (Hosmer et al., 2013). The internal validation yielded an optimism of less than 0.01 for the final model. Hence, these results demonstrate that the final model had a validated estimate of predictive accuracy and had excellent discrimination for predicting EBTSCC violations $>400,000$ cells $/ \mathrm{mL}$. The same methodology was applied to the full model before it was reduced by a backward selection procedure. The full model had similar optimism, thereby offering greater confidence that the discriminatory ability of the final model was not compromised relative to the full set of variables evaluated (Efron and Tibshirani, 1993).

The optimum cut-point of the ROC curve for distinguishing between a predicted likely violation and a nonviolation was 0.12 . This can be interpreted as any herd that had a probability of an EBTSCC threshold violation $>0.12$ was expected to exceed the threshold at the next test-month. This cut-point corresponds to the prevalence of the EBTSCC threshold violation in the study database, and is the point where the Se and Sp of the model are optimized at their joint maximum values. The low PPV and high NPV of the model resulted in low accuracy of prediction for herds that would violate the EBTSCC threshold but very good prediction of herds that would not. Herds with monitored variables indicating high risk may not actually experience a violation, whereas herds with variables indicating a low risk were very unlikely to experience a violation. This is largely a function of the relatively low prevalence of herds that experience the threshold violation in the study population.

The shape of the ROC curve suggests that the sensitivity of the model for predicting EBTSCC violations varies greatly. In contrast, the narrow band of high $\mathrm{Sp}$ values is consistent with the observation that the model is better calibrated for predictions at the lower end of the expected risk scale. This stands to reason from both a statistical perspective and a subject matter perspective. Statistically, the number of observations used to estimate the model was much greater for the segment of the study population that did not experience a violation of EBTSCC than for those that did. Again, from a subject matter perspective, it may be that there were many different effective risk profiles for high versus low EBTSCC. High EBTSCC profiles would have been characterized by different combinations of components contributing to high EBTSCC, whereas low EBTSCC profiles would have been simpler and had individual risk factors that were consistent with low EBTSCC. It would be interesting to test this hypothesis in future research.

As discussed previously, some variation may exist between the EBTSCC reported here and the actual BTSCC that would have been realized. Dairy producers had access to their weekly BTSCC and, accordingly, could have implemented important management decision based on those results. For example, a producer that had a BTSCC $>400,000$ cells $/ \mathrm{mL}$ in the first 2 BTSCC tests of the month would have had the opportunity to cull or dry-off cows with high SCC in the current month. In turn, this could have reduced the BTSCC to nonviolative levels, whereas the final model would have predicted an EBTSCC exceeding 400,000 cells/mL. In addition, several events other than management interventions could have caused a divergence between EBTSCC and actual BTSCC. Calving cows, illness, herd health visit, feeding modifications, staff changes, and weather changes could have influenced the next month's BTSCC and affected the accuracy of the prediction model. 
It is noteworthy that the database used for the construction of the prediction model contained 2007 and 2008 DHI data. In those years, the Canadian Dairy Commission mandated that the threshold limit for BTSCC violations was 500,000 cells/mL (Canadian Dairy Commission, 2013). The prediction model was constructed to predict a violation using the current cutoff of 400,000 cells $/ \mathrm{mL}$. The change in the violation threshold may have affected the accuracy of the model because dairy producers would have likely taken more aggressive actions toward managing BTSCC if the violation limit had been at 400,000 cells/mL during 2008. To improve the prediction model, it would be useful to perform an external validation of the model using a database constructed with data collected from August 2012 onward, after the new violation threshold of 400,000 cells/mL was adopted.

The model was constructed using Québec data and was intended for use by dairy practitioners and advisors in Québec, Canada. However, this model could be of interest to dairy herds and advisors outside Québec. Before this model is used in a different population, however, it is important to consider some factors that could affect the variables included in the model, and consequently, the prediction accuracy. For example, the mean number of milking cows per herd is greater in several other Canadian provinces than that in the present study (mean of 55 cows, range 30 to 511 cows; CanWest DHI, 2008). The relative contribution of each cow to the BTSCC would be smaller in larger herds and greater in smaller herds (Lukas at al., 2008). Consequently, the small changes in some of the indices from one month to the next in larger herds could improve model accuracy. Daily milk yield per cow is greater in the western Canadian provinces than it is in Québec (CanWest DHI, 2008). This would also affect the accuracy of the model because the dilution effect could improve the stability of the BTSCC and increase the accuracy of model prediction. Mastitis pathogens that are present in a herd and the relative prevalence of contagious mastitis pathogens could also influence the model. Staphylococcus aureus IMI, which usually cause chronically elevated SCC in infected cows, are more prevalent in Québec dairy herds than in herds in several other Canadian provinces (Olde Riekerink at al., 2010).

\section{Practical Utility of the Model}

Results of this study can easily be used by dairy veterinarians to advise their clients of the likelihood of a possible impending BTSCC violation. For example, it is possible to compare the predicted probabilities estimated for 5 hypothetical herd situations. These 5 herds would represent the 10th, 25th, 50th, 75th, and 90th percentile categories for all 6 variables included in the model. A hypothetical herd with the value of each index corresponding to the 90th percentile would have a probability of 0.04 of exceeding an EBTSCC of 400,000 cells $/ \mathrm{mL}$ in the next month. This probability increases to 0.52 in herds corresponding to the 10th percentile. Herds at 75 th, 50th, and 25th percentiles would have probabilities of $0.09,0.18$, and 0.34 , respectively, of an EBTSCC 400,000 cells/mL violation. Managers of herds with a probability greater than 0.18 (which represents the probability of a herd presenting with median variable values) should consider this information seriously and implement intervention strategies to improve udder health status within herd. In addition, the level of intervention could be modified between herds depending on the each herd's objective and vision of udder health.

\section{CONCLUSIONS}

General herd udder health status is important for maintaining BTSCC under a target level. Active monitoring of mean individual SCS and the proportion of cows with $\mathrm{SCC}>500,000$ cells $/ \mathrm{mL}$ at the current test could help to reduce the likelihood of a BTSCC violation at the new 400,000 cells/mL threshold. An internally valid logistic regression model combines these variables into a BTSCC violation risk index for simplicity of application. The prediction model constructed in this study is a potentially good tool for dairy veterinarians to advise their clients of the possibility of an upcoming BTSCC violation.

\section{REFERENCES}

Ali, A. K. A., and G. E. Shook. 1980. An optimum transformation for somatic cell concentration in milk. J. Dairy Sci. 63:487-490.

Avalos, M. 2009. Sélection de variables avec lasso dans la régression logistique conditionnelle. In 41èmes Journées de Statistique, SFdS, Bordeaux, Bordeaux, France.

Bates, D., M. Maechler, B. M. Bolker, and S. Walker. 2014. lme4: Fitting linear mixed-effects models using lme4. Accessed Jan. 29, 2015. http://arxiv.org/abs/1406.5823. Submitted to Journal of Statistical Software.

Canadian Dairy Commission. 2013. Canadian Dairy Commission. 2013. Mandate. Accessed Jan. 29, 2015. http://www.cdc-ccl.gc.ca/ CDC/index.php.

CanWest DHI. 2008. Progress report. Accessed Oct. 27, 2014. http:// www.canwestdhi.com/pdf_files/2008\%20progress\%20report\%20 statistics.pdf.

Cox, D. R. 1958. Two further applications of a model for binary regression. Biometrika 45:562-565.

Dohoo, I. R., and K. E. Leslie. 1991. Evaluation of changes in somatic cell counts as indicators of new intramammary infections. Prev. Vet. Med. 10:225-237.

Efron, B., and R. J. Tibshirani. 1993. An Introduction to the Bootstrap. Chapman \& Hall/CRC Press, Boca Raton, FL.

Fauteux, V., J. P. Roy, D. T. Scholl, and E. Bouchard. 2014. Benchmarks for evaluation and comparison of udder health status using monthly individual somatic cell count. Can. Vet. J. 55:741-748. 
Friedman, J., T. Hastie, and R. Tibshirani. 2010. Regularization paths for generalized linear models via coordinate descent. J. Stat. Softw. 33:1-22. http://www.jstatsoft.org/v33/i01/. Accessed Oct. $27,2014$.

Hand, K. J., M. A. Godkin, and D. F. Kelton. 2012. Short communication: Bulk milk somatic cell penalties in herds enrolled in Dairy Herd Improvement programs. J. Dairy Sci. 95:240-242.

Harrell, F. E. 2001. Regression Modeling Strategies: With Applications to Linear Models, Logistic Regression, and Survival Analysis. Springer, New York, NY.

Harrell, F. E., K. L. Lee, and D. B. Mark. 1996. Tutorial in biostatistics-Multivariable prognostic models: Issues in developing models, evaluating assumptions and adequacy, and measuring and reducing errors. Stat. Med. 15:361-387.

Hosmer, D. W., J. S. Lemeshow, and R. X. Sturdivant. 2013. Applied Logistic Regression. 3rd ed. Wiley Series in Probability and Statistics. Wiley Intersciences, Hoboken, NJ.

Hosmer, D. W., and S. Lemeshow. 2000. Applied Logistic Regression. 2nd ed. Wiley Intersciences, Hoboken, NJ.

Husson, F., J. Josse, S. Le, and J. Mazet. 2014. FactoMineR: Multivariate exploratory data analysis and data mining with $\mathrm{R}$. $\mathrm{R}$ package version 1.26. Accessed Oct. 27, 2014. http://cran.r-project. org $/$ package $=$ FactoMineR .

Joliffe, I. T. 1972. Discarding variables in a principal component analysis. I: Artificial data. J. R. Stat. Soc. Ser. C Appl. Stat. 21:160-173.

Joliffe, I. T., and B. J. T. Morgan. 1992. Principal component analysis and exploratory factor analysis. Stat. Methods Med. Res. 1:69-95.

Krzanowski, W. J. 1987. Selection of variables to preserve multivariate data structure, using principal components. J. R. Stat. Soc. Ser. C Appl. Stat. 36:22-33.

Les Producteurs de Lait du Québec. 2009. 2008 Annual report. Accessed Oct. 27, 2014. http://www.lait.org/lait_files/File/ decouvrir_production/rapport/RA2008.pdf.

Les Producteurs de Lait du Québec. 2013. Milk quality criteria. Accessed Oct. 27, 2014. https://www.fplq.qc.ca/Bibliotheque/ Brochure/Criteres_Qualite_ANG.pdf.
Les Producteurs de Lait du Québec. 2014. 2013 Annual report-Quality control. Accessed Oct. 27, 2014. http://www.lait.org/fichiers/ RapportAnnuel/FPLQ-2013/controle_qual.pdf.

Lievaart, J. J., H. W. Barkema, J. van den Broek, J. A. P. Heesterbeek, and W. D. J. Kremer. 2010. Prediction of the herd somatic cell count of the following month using a linear mixed effect model. J. Dairy Sci. 93:234-241.

Lievaart, J. J., W. D. J. Kremer, and H. W. Barkema. 2007. Short communication: Comparison of bulk milk, yield-corrected and average somatic cell counts as parameters to summarize the subclinical mastitis situation in a dairy herd. J. Dairy Sci. 90:4145-4148.

Lukas, J. M., J. K. Reneau, and M. L. Kinsel. 2008. Predicting somatic cell count standard violations based on herd's bulk tank somatic cell count. Part 1: Analysing variation. J. Dairy Sci. 91:427-432.

Madouasse, A., J. N. Huxley, W. J. Browne, A. J. Bradley, and M. J. Green. 2010. Somatic cell count dynamics in a large sample of dairy herds in England and Wales. Prev. Vet. Med. 96:56-64.

McCabe, G. P. 1984. Principal variables. Technometrics 26:137-144.

Miller, M. E., C. D. Langefeld, W. M. Tierney, S. L. Hui, and C. J. McDonald. 1993. Validation of probabilistic predictions. Med. Decis. Making 13:49-58.

Miller, M. E., L. H. Siu, and M. William. Tierney. 1991. Validation techniques for logistic regression models. Stat. Med. 10:12131226.

Olde Riekerink, R. G. M., H. W. Barkema, D. T. Scholl, D. E. Poole, and D. F. Kelton. 2010. Management practices associated with the bulk-milk prevalence of Staphylococcus aureus in Canadian dairy farms. Prev. Vet. Med. 97:20-28.

R Core Team. 2012. R: A language and environment for statistical computing. R Foundation for Statistical Computing, Vienna. Austria.

Steyerberg, E. W., F. E. Harrell Jr., G. J. J. M. Borsboom, M. J. C. Eijkemans, Y. Vergouwe, and J. D. F. Habbema. 2001. Internal validation of predictive models: Efficiency of some procedures for logistic regression analysis. J. Clin. Epidemiol. 54:774-781.

Tibshirani, R. 1996. Regression shrinkage and selection via the lasso. J. R. Stat. Soc. Ser. B Stat. Methodol. 58:267-288. 